\title{
Identification of differentially expressed micro-RNA in rotator cuff tendinopathy
}

\author{
Kimberly E. Hall ${ }^{1}$ \\ Eric J. Sarkissian ${ }^{1}$ \\ Orr Sharpe 2,3 \\ William H. Robinson ${ }^{2,3}$ \\ Geoffrey D. Abrams ${ }^{1,3}$
}

1 Stanford University School of Medicine, Department of Orthopedic Surgery, Stanford (CA), USA

2 Stanford University School of Medicine, Department of Medicine, Division of Rheumatology and Immunology, Stanford (CA), USA

3 Veterans Administration, Palo Alto, Palo Alto (CA), USA

Corresponding author:

Geoffrey D. Abrams

Department of Orthopedic Surgery,

Stanford University

Veterans Administration - Palo Alto

341 Galvez Street

94035 Stanford (CA), USA

E-mail: gabrams@stanford.edu

\section{Summary}

Introduction. The pathomolecular events behind the development of tendinopathy remain unknown.

Methods. Paired biopsies from human shoulder supraspinatus (tendinopathy) and subscapularis (control) tendons were obtained from patients undergoing shoulder arthroscopy, with tendinopathy confirmed using light microscopy evaluation. Differentially expressed micro-RNA were identified using RNA sequencing analysis, with target genes for the differentially expressed micro-RNA predicted using miRbase and Targetscan. The micro-RNA were secondarily screened to identify those with predicted binding to genes known be involved in tendinopathy - collagen 1A1/1A2/3A1, BMP-2/7, and IL-6 - with additional cross referencing of gene/micro-RNA predicted binding performed with NetworkAnalyst.

Results. Supraspinatus samples demonstrated significantly greater histologic characteristics of tendinopathy versus subscapularis samples. A total of 21 micro-RNA were differentially expressed between normal and diseased tendon, with six (let-7g, miR-7a, miR-22, miR-26a/b, and
miR-29a) having predicted binding to genes known to be involved in tendinopathy.

Conclusion. The identiied micro-RNAs may be involved in the development of tendon disease and represent potential therapeutic targets.

Level of evidence: Basic science investigation.

KEY WORDS: tendinopathy, micro-RNA, inflammation, rotator cuff, tendon, shoulder.

\section{Introduction}

Tendinopathy is physically disabling, leads to pain with physical activity, and results in an estimated $\$ 30$ billion dollars in treatment costs annually in the United States ${ }^{1}$. Of the nearly 100 million office visits for musculoskeletal complaints in $2010^{2}$, approximately $30 \%$ were related to tendinopathy diagnoses ${ }^{3}$. Even though treatment can extend beyond six months, $30 \%$ of patients never return to prior levels of physical activity, underscoring the need for better therapeutic strategies.

Collagen accounts for approximately $85-90 \%$ of the dry mass of non-pathologic tendon, of which $98 \%$ is type I collagen (Col1). Within normal tendon, parallel arrays of predominantly Col1 are aligned in a regular linear pattern with tenocytes interspersed among the collagen, with cell and collagen turnover closely regulated. In diseased tendons, however, degeneration and disorganization of collagen fibers occur ${ }^{4}$ and there is an increase in catabolic inflammatory mediators $^{5-7}$. In addition, tenocytes undergo a phenotypic change $^{6}$ and type III collagen (Col3) within the extracellular matrix is increased ${ }^{8}$. The mechanism for these changes remains poorly understood, however, there is evidence that increased bone morphogenic protein (BMP) expression in diseased tendon may impair tenocyte differentiation from resident tendon stem progenitor cells (TSPC) $)^{9-11}$.

Micro-RNA (miRNA/miR) are small non-coding RNAs that regulate post-transcriptional gene expression by binding to the $3^{\prime}$ UTR target sites of messenger RNA (mRNA), thus inhibiting translation through a RNA-induced silencing complex (RISC) ${ }^{12}$. They have been shown to be involved in a wide range of biological processes, including stem cell differentiation ${ }^{13-15}$. In addition, they have demonstrated regulatory function over inflammatory mediator production ${ }^{16-20}$ as well as collagen ${ }^{12,13,19,21}$ and BMP expression ${ }^{10}$, all of which are associated with tendon disease. Because of this, they remain an attractive target for therapeutic inter- 
vention and warrant further investigation into their role in the pathogenesis of tendinopathy.

The differential expression of miRNA within rotator cuff tendinopathy has yet to be described. Understanding which micro-RNA are up- and down-regulated within tendinopathy may help guide identification of key miRNA within the disease process. Using miRNA sequencing, we sought to identify differentially expressed miRNA within normal and diseased tendon as well as further screen for miRNA predicted to exert regulatory control over genes which regulate tendon homeostasis.

\section{Materials and methods}

Institutional Review Board (IRB) approval and patient consent was obtained prior to conducting the investigation. All method comply with the ethical standards of Muscle, Ligaments, Tendon Journal as previously described $^{22}$. Five male patients consented to have paired supraspinatus (SS) and subscapularis (SC) tendon tissue biopsies taken while undergoing arthroscopic shoulder surgery. Inclusion criteria were age 18 years and older with a history and exam consistent with shoulder pain, partial thickness tearing and tendinopathy of the supraspinatus tendon as determined by magnetic resonance imaging (MRI) and direct arthroscopic visualization, and no tearing or tendinopathy of the subscapularis on MRI or arthroscopic visualization. Exclusion criteria included history of systemic or musculoskeletal inflammatory disease, a diagnosis of diabetes or hypercholesterolemia requiring medication, current smoking habit, receiving a corticosteroid injection to the operative shoulder within three months of surgery, or oral antiinflammatory medication within two weeks of surgery. Biopsies were obtained from the articular side of the supraspinatus and from the superolateral portion of the subscapularis just adjacent to its insertion. The specimens for histologic analysis were frozen at $80^{\circ} \mathrm{C}$ in optimum cutting temperature compound (TissueTek, Torrance, CA, USA), and 10- $\mu$ m cryosections were cut and affixed on glass slides. Sections from three different depths of the sample were used to obtain a representative sample of the entire specimen. Tissue was then stained with hematoxylin and counterstained with eosin. Histologic grading under light microscopy was measured using the Bonar scoring system ${ }^{23}$ with two observers' scores averaged.

The other set of samples were placed in RNA later and frozen in liquid nitrogen. Micro-RNA was purified using a commercially available kit (Qiagen miRNeasy Mini Kit) according to the manufacturer protocol. RNA integrity and quantitation analysis was performed using the 2100 Bioanalyzer and the RNA Pico quantitation method (Agilent Technologies Inc, Santa Clara, CA, USA). The NEBNext ${ }^{\circledR}$ Multiplex Small RNA Library Prep Set for Illumina ${ }^{\circledR}$ (New England Biolabs, Ipswich, MA, USA) was used for small RNA library preparation and high throughput sequencing was conducted using the HiSeq 2500 (1x150) (Illumina ${ }^{\circledR}$, San Diego, CA, USA). To perform sequence analysis, Flow, v3.0 (Partek ${ }^{\circledR}$ Incorporated, St. Louis, Missouri, USA) was utilized with miRbase v.20 for alignment and annotation. Seed mismatch of one was allowed to increase validity. Normalization of reads was done using the reads per million (RPM) formula: (read counts of an individual miRNA/sum of read counts of all mappable miRNAs) multiplied by $1 \times 10^{\wedge} 6$.

\section{Genetic analysis}

We cross-referenced our differentially expressed miRNA with those miRNA predicted to have post-translational regulatory control over genes known to have altered expression in tendinopathic samples ${ }^{4}, 11,24-26$ (Tab. I) using miRbase and Targetscan. To identify any possible missed associations or predicted binding, we performed the reverse analysis (inputting gene instead of miRNA) using NetworkAnalyst, a visual analytics tool for gene expression profiling $27,28$. The genes COL1A1, COL1A2, COL3A1, BMP2, BMP7, and IL-6 (Tab. I) were used as input for NetworkAnalyst and the individual miRNA which were identified as potential mediators of gene expression over the identified targets were compared for similar miRNA to our differential expression data.

\section{Statistical analysis}

Individual RPMs were averaged among patients to determine total number of miRNAs present while comparisons between patients were performed using a paired Student's $t$-test. Bonar scoring was compared with a paired Student's $t$-testing with intra-observer correlation measures using Cohen's kappa coefficient. An alpha value of 0.05 was set as significant.

\section{Results}

Ten paired samples were collected from five male patients (average age 58 years, range 44-65 years). All patients underwent shoulder arthroscopy with debridement and subacromial decompression, with four patients additionally undergoing subpectoral biceps tenodesis and one patient undergoing combined subpectoral bicep tenodesis and arthroscopic distal clavi-

Table I. Gene symbol and names of genes known to be dysregulated within tendinopathy.

\begin{tabular}{ll}
\hline Gene Symbol & Gene Name \\
\hline COL1A1 & Collagen type 1 alpha 1 chain \\
COL1A2 & Collagen type 1 alpha 2 chain \\
COL3A1 & Collagen type 3 alpha 1 chain \\
BMP2 & Bone morphogenic protein 2 \\
BMP7 & Bone morphogenic protein 7 \\
IL-6 & Interleukin-6 \\
\hline
\end{tabular}



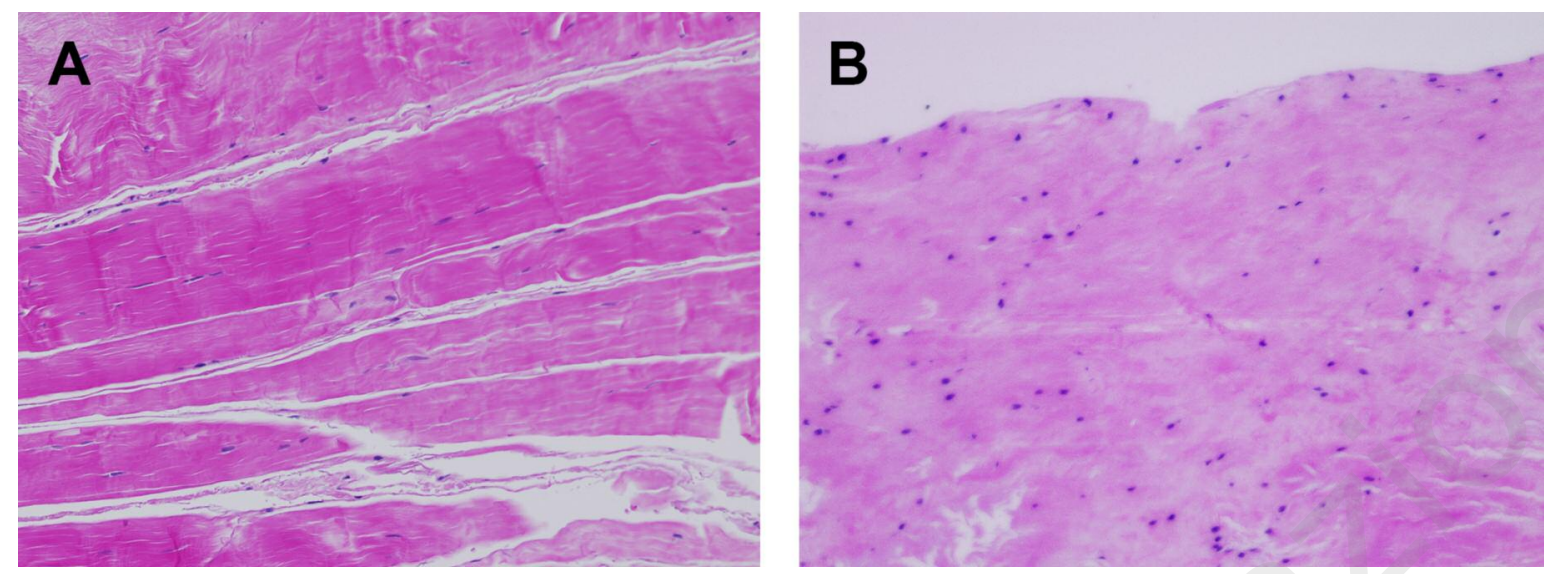

Figure 1. Light microscopy image (20x) of (A) subscapularis and (B) supraspinatus tendon samples. Note the disorganization and increased cellularity within the supraspinatus sample, indicating tendinopathy.

cle resection. The average Bonar score for supraspinatus was $1.6+/-0.5$ and subscapularis was 0.4 +/- 0.5 ( $p=0.03$; $k=0.73$ ) (Fig. 1).

RNA samples had no significant degradation and all were included in the analysis. A total of 633 miRNAs were identified in supraspinatus tendon samples, with 346 miRNAs having at least one RPM. For the subscapularis tendon, a total of 718 miRNA were identified, with 381 having an average RPM $>1$. In the paired analysis, only 21 miRNAs were differentially expressed between supraspinatus and subscapularis, with a decreased RPM in the diseased state (supraspinatus) in all but six of the miRNA (Tab. II).

Cross referencing the identified differentially expressed miRNA in our samples with those predicted to play a role in post-translational gene regulation for the identified genes resulted in six matching miRNAs (Tab. III). The NetworkAnalyst prediction of key micro-RNA for the genes in question is shown in Figure 2.

\section{Discussion}

In comparing miRNA expression patterns between supraspinatus and subscapularis, we found $21 \mathrm{miR}$ NAs were significantly differentially expressed, with a majority demonstrating decreased expression in the diseased state. Of these miRNA, six were predicted to have regulatory control over genes known to be dysregulated in tendinopathy.

Micro-RNA, given its role in post-transcriptional regulation, has the ability to affect expression of both cytokines as well as collagen transcripts ${ }^{29}$, both known to be hallmarks of tendinopathy. Many miRNA have been identified in the literature to have such a role. MiR-23a and miR-26a, both of which were identified in this investigation as having decreased expression in tendinopathic tissue, have been shown to regulate IL-6 ${ }^{30}$ as well as other pro-inflammatory cytokines such as TNF- $a^{31}, 32$. Such down-regulation of microRNA, as demonstrated in our investigation within pathologic samples, may allow for increased inflam- matory mediator production. Interestingly, the interactions between miR and inflammatory mediators may be bi-directional, with Zhang et al. reporting IL-6 upregulation correlated to decreased expression of miR$26 \mathrm{a}^{33}$. There are also numerous examples of miRNA exerting regulatory control over collagen expression. Millar et al. demonstrated that miR-29a, through IL33 , leads to changes in the collagen matrix in a model of early tendinopathy ${ }^{19}$. They observed that downregulation of miR-29a allowed for increased amounts of Col3 production. Like our study, they found that miR-29a was differentially expressed in early tendinopathic samples, with a nearly two-fold expression decrease in miRNA in the pathologic tissue as opposed to the control. Micro RNA-29 has also been shown to regulate other collagen types, including Col1 in the eye $^{34,35}$, cardiac fibroblasts ${ }^{36}$, and hepatocytes ${ }^{37}$.

While there have been no reports describing association of miRNA and BMP levels within a tendon model, $\mathrm{miR}$ has been shown to have regulatory control over BMP in other disease states. This is significant as increased expression of BMP has been shown to impair the development of tendon stem progenitor cells into tenocytes ${ }^{9,38}$, a critical cellular component of native tendon tissue. Icli et al., investigating angiogenesis from endothelial cells, reported that systemic intravenous administration of an miR-26a inhibitor increased BMP expression and rapidly induced robust angiogenesis ${ }^{10}$. In addition, miR-22 and BMP-7 interactions have been studied within renal tubular cells. Using a unilateral renal obstruction model of kidney fibrosis, Long et al. reported that renal fibroblast from miR-22-deficient mice demonstrated a significant increase in BMP-7 expression ${ }^{39}$. This phenotype could be reversed when cells were transfected with miR-22 mimics.

The role of micro-RNA in the development and differentiation of stem cells continues to evolve, with recent hypotheses centering around distinct miRNAs mediating stem cell renewal and differentiation in specific cell types and at precise cellular development stages. For example, miR-22 and miR-29, both 
Table II. Differential expression of miRNA between supraspinatus and subscapularis using micro-RNA sequencing analysis.

\begin{tabular}{|c|c|c|c|}
\hline Micro-RNA & p-value & Decreased Expression & Expression Change \\
\hline hsa-miR-199b-5p & 0.001 & Supra & 1.75 \\
\hline hsa-miR-140-5p & 0.001 & Subscap & 0.54 \\
\hline hsa-miR-26a-5p & 0.001 & Supra & 1.29 \\
\hline hsa-miR-532-5p & 0.001 & Supra & 1.98 \\
\hline hsa-miR-199a-5p & 0.003 & Supra & 1.66 \\
\hline hsa-miR-222-3p & 0.003 & Subscap & 0.43 \\
\hline hsa-miR-29a-3p & 0.005 & Supra & 1.67 \\
\hline hsa-miR-92a-3p & 0.006 & Supra & 1.67 \\
\hline hsa-miR-22-3p & 0.006 & Supra & 1.35 \\
\hline hsa-let-7e-5p & 0.011 & Subscap & 0.45 \\
\hline hsa-miR-191-5p & 0.014 & Supra & 1.26 \\
\hline hsa-miR-10a-5p & 0.014 & Supra & 3.24 \\
\hline hsa-miR-199b-3p & 0.015 & Supra & 1.93 \\
\hline hsa-miR-199a-3p & 0.015 & Supra & 1.93 \\
\hline hsa-miR-126-3p & 0.023 & Supra & 10.70 \\
\hline hsa-let-7i-5p & 0.029 & Supra & 1.44 \\
\hline hsa-miR-30c-5p & 0.030 & Supra & 1.91 \\
\hline hsa-let-7g-5p & 0.039 & Supra & 1.45 \\
\hline hsa-miR-30d-5p & 0.044 & Supra & 1.42 \\
\hline hsa-miR-100-5p & 0.049 & Subscap & 0.55 \\
\hline hsa-miR-151a-3p & 0.050 & Supra & 1.57 \\
\hline hsa-miR-127-3p & 0.051 & Supra & 1.92 \\
\hline hsa-miR-7a-5p & 0.056 & Subscap & 0.66 \\
\hline hsa-miR-26b-5p & 0.062 & Supra & 1.89 \\
\hline hsa-miR-25-3p & 0.064 & Supra & 1.41 \\
\hline hsa-miR-99a-5p & 0.071 & Supra & 1.95 \\
\hline hsa-miR-125a-5p & 0.080 & Subscap & 0.58 \\
\hline hsa-miR-30a-5p & 0.088 & Supra & 3.55 \\
\hline hsa-miR-23a-3p & 0.092 & Supra & 1.91 \\
\hline
\end{tabular}

Table III. Micro-RNAs in common between differentially expressed miRNA in supraspinatus/subscapularis tendon samples and miRNAs predicted to have posttranslational regulatory control over COL1A1, COL1A2, COL3A1, BMP2, BMP7, and IL6.

\begin{tabular}{lll}
\hline hsa-miR-7a-5p & hsa-miR-22-3p & hsa-miR-29a-3p \\
hsa-let-7g-5p & hsa-miR-26a-5p & hsa-miR-26b-5p \\
\hline
\end{tabular}

miRNA found to be differentially regulated in this investigation, play a role in differentiation of embryonic stem cells ${ }^{40,41}$. Furthermore, miR-23, miR-26, and miR-29 have also been implicated in the development of astrocytes in neuronal stem cell differentiation ${ }^{42}$. Micro-RNA-26 has also been found to prevent the differentiation of mesenchymal stem cells into osteoblasts by downregulating specific transcription factors required for osteoblast differentiation ${ }^{15}$. 


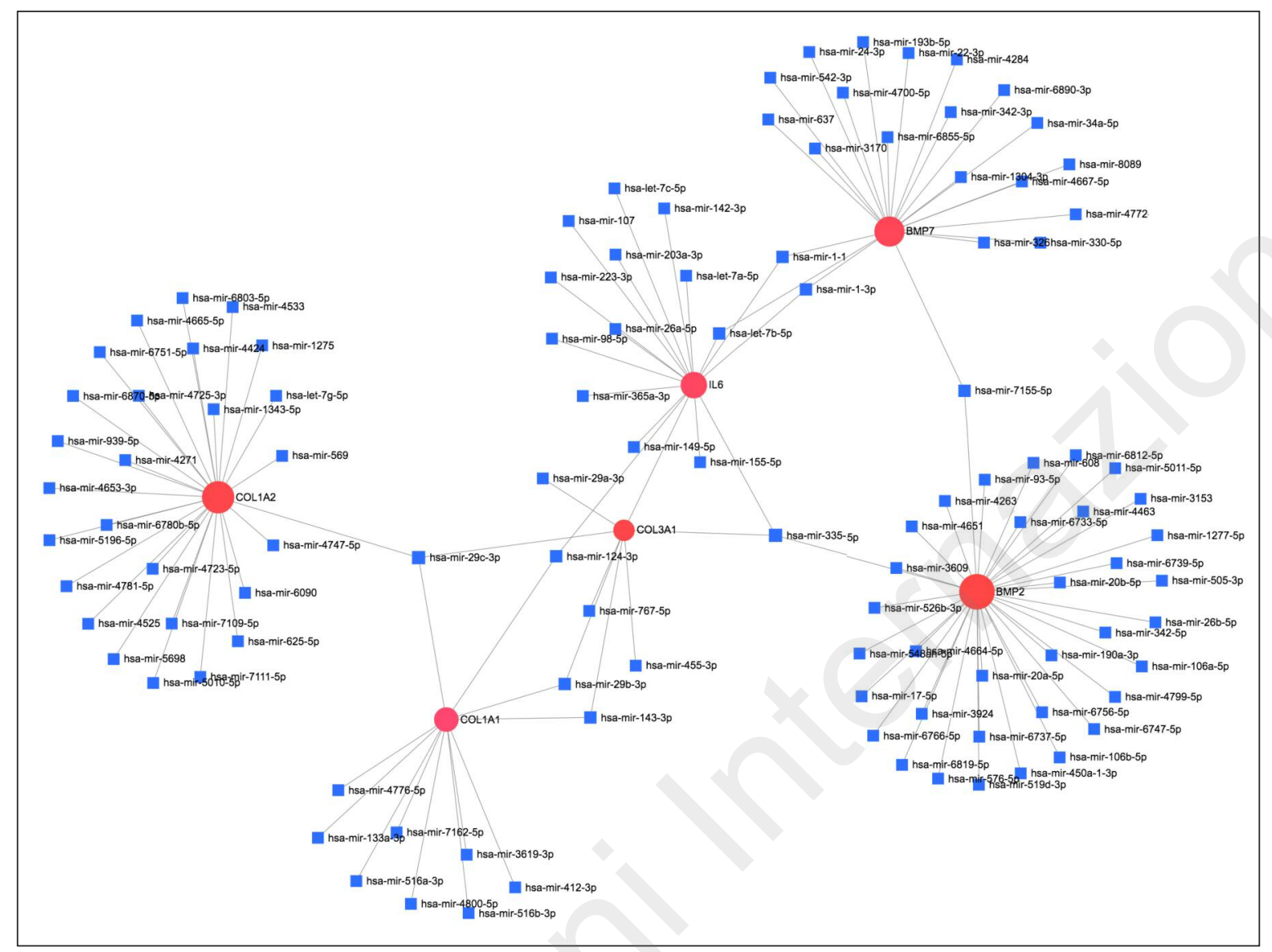

Figure 2. Network interaction diagram demonstrating miRNA associated with identified genes.

One of the only other studies to determine differential miRNA expression in tendinopathy was performed by Thankam et al., who examined miRNA expression profiles of the long head of the biceps tendon in patients with and without shoulder osteoarthritis using microarray analysis ${ }^{43}, 44$. They sought to determine epigenetic pathways which mediate inflammation within the shoulder through Janus kinase (JAK)/signal transducers and activators of transcription-3 (STAT) pathway, which is a key regulator of inflammatory mediator expression 43 . They reported significant down-regulation of 196 miRNA and up-regulation of 39 miRNA $^{43}$. Highly down-regulated miRNAs included hsa-miR-191-5p, hsa-miR-361-5p, hsa-miR-1273 g3p, hsa-miR-99b-5p, hsa-miR-145-5p, hsa-miR-99a$5 p$, and hsa-miR-100-5p while up-regulated ones included hsa-miR-4467, hsa-miR-6723-5p, hsa-miR8071 , and hsa-miR-5001-5p 43 . While a number of miRNA identified in their investigation overlap with our findings, there are some important methodological differences between studies. First, the specimens used by Thankam et al. ${ }^{43}$ were long head of the biceps tendons in different patients versus paired supraspinatus and subscapularis tendon samples in our study. Furthermore, the disease states varied between patients in the current study and Thankam et al. ${ }^{43}$ This investigation sought to determine miRNAs which are differentially regulated early in the disease process, hence biopsies of tendinopathic supraspinatus tendons without long standing full-thickness tearing pathology. In contrast, Thankam et al. ${ }^{43}$ utilized biceps tendons from patients without arthritis and compared them to patients with severe glenohumeral arthritis. Additionally, Thankam et al. ${ }^{43}$ used micro-array analysis while we utilized RNA-sequencing techniques. Although micro-array analysis can provide for a large number of targets, RNA-sequencing gives the investigators access to the entire transcriptome.

In addition to modulation of collagen and inflammatory mediator gene expression, the information presented here suggests the possibility of a new model in the pathogenesis of tendinopathy: modulation of tendon stem progenitor cells by micro-RNA. It is clear from the literature that tendinopathy is associated with molecular inflammation and the production of inflammatory mediators. Furthermore, these inflammatory mediators have been shown to influence the expression of micro-RNA, which in turn may lead to altered expression of the inflammatory mediators themselves as well as modulate differentiation of resident stem cells within tendon tissue. For example, with an inflammatory-mediated decrease in micro-RNA expression, osteoblastic differentiation may be favored over tenocyte development from TSPCs, leading to the 
collagen and eventual biomechanical alterations found in tendinopathy.

There were some limitations to our study. Mainly, with only ten samples from five male individuals, our low sample size and use of a single gender may not reflect the proteomic profile of a larger population. We did find differential expression of a number of miRNAs in this population, but averaged over a larger group, it is possible that other miRNAs may have shown significant differential expression. Furthermore, we limited our secondary analysis to only six genes known to be involved in tendinopathy. Including others, may have produced more targets in the final analysis, but inclusion of these additional genes would not change the raw differential expression data presented in Table II. Lastly, sampling error during biopsy of the tendon tissue may have lead to aberrant results. This limitation was mitigated, however, by the use of light microscopy to confirm normal versus diseased tendon within the samples.

The data presented here suggest specific microRNA which may be involved in the development of tendinopathy. Given the central regulatory role of microRNA over gene expression, this raises the possibility of providing exogenous microRNA analogues or microRNA inhibitors in order to modulate the development of tendinopathy in humans. This would serve to affect both inflammatory mediator production as well as collagen changes, both of which are known to occur in the development of tendinopathy. One of the only studies to investigate this possibility examined the effect of equine tendon damage with an without exogenous microRNA 29a. The Authors reported that locally injected microRNA-29a facilitated tissue remodeling in an equine tendinopathy model ${ }^{45}$.

\section{Conclusion}

The identified micro-RNAs may be involved in the development of tendon disease and represent potential therapeutic targets.

\section{Conflict of interest}

No other relationships/conditions/circumstances that present potential conflict of interest.

\section{References}

1. Chen J, Xu J, Wang A, Zheng M. Scaffolds for tendon and ligament repair: review of the efficacy of commercial products. Expert Rev Med Devices. 2009;6(1):61-73.

2. National Ambulatory Medical Care Survey. Centers for Disease Control and National Center for Health Statistics. 2010.

3. Scott A, Ashe MC. Common tendinopathies in the upper and lower extremities. Curr Sports Med Rep. 2006;5(5):233-241.

4. Xu Y, Murrell GA. The basic science of tendinopathy. Clin Orthop Relat Res. 2008;466(7):1528-1538.

5. Al-Sadi O, Schulze-Tanzil G, Kohl B, Lohan A, Lemke M, Ertel
W, et al. Tenocytes, pro-inflammatory cytokines and leukocytes: a relationship? Muscles Ligaments Tendons J. 2011;1 (3):68-76.

6. Archambault JM, Jelinsky SA, Lake SP, Hill AA, Glaser DL, Soslowsky LJ. Rat supraspinatus tendon expresses cartilage markers with overuse. J Orthop Res. 2007;25(5):617-624.

7. Blomgran P, Blomgran R, Ernerudh J, Aspenberg P. Cox-2 inhibition and the composition of inflammatory cell populations during early and mid-time tendon healing. Muscles Ligaments Tendons J. 2017;7(2):223-229.

8. de Mos M, van El B, DeGroot J, Jahr H, van Schie HT, van Arkel ER, et al. Achilles tendinosis: changes in biochemical composition and collagen turnover rate. Am J Sports Med. 2007;35(9):1549-156.

9. Bi Y, Ehirchiou D, Kilts TM, Inkson CA, Embree MC, Sonoyama W, et al. Identification of tendon stem/progenitor cells and the role of the extracellular matrix in their niche. Nat Med. 2007;13(10):1219-1227.

10. Icli B, Wara AK, Moslehi J, Sun X, Plovie E, Cahill M, et al. MicroRNA-26a regulates pathological and physiological angiogenesis by targeting BMP/SMAD1 signaling. Circ Res. 2013;113(11):1231-1241.

11. Lui PP. Histopathological changes in tendinopathy: potential roles of BMPs? Rheumatology (Oxford). 2013;52(12):21162126.

12. Ambros $V$. The functions of animal microRNAs. Nature. 2004;431(7006):350-355.

13. Chen L, Wang GD, Liu JP, Wang HS, Liu XM, Wang Q, et al. miR-135a modulates tendon stem/progenitor cell senescence via suppressing ROCK1. Bone. 2015;71:210-216.

14. Gangaraju VK, Lin H. MicroRNAs: key regulators of stem cells. Nat Rev Mol Cell Biol. 2009;10(2):116-125.

15. Luzi E, Marini F, Sala SC, Tognarini I, Galli G, Brandi ML. Osteogenic differentiation of human adipose tissue-derived stem cells is modulated by the miR-26a targeting of the SMAD1 transcription factor. J Bone Miner Res. 2008;23(2):287-295.

16. Chen $\mathrm{CH}$, Zhou YL, Wu YF, Cao Y, Gao JS, Tang JB. Effectiveness of microRNA in down-regulation of TGF-beta gene expression in digital flexor tendons of chickens: in vitro and in vivo study. J Hand Surg Am. 2009;34(10):1777-1784 e1.

17. Hao M, Zhang L, An G, Sui W, Yu Z, Zou D, et al. Suppressing miRNA-15a/-16 expression by interleukin- 6 enhances drugresistance in myeloma cells. J Hematol Oncol. 2011;4:37.

18. Mendias CL, Gumucio JP, Lynch EB. Mechanical loading and TGF-beta change the expression of multiple miRNAs in tendon fibroblasts. J Appl Physiol (1985). 2012;113(1):56-62.

19. Millar NL, Gilchrist DS, Akbar M, Reilly JH, Kerr SC, Campbell $\mathrm{AL}$, et al. MicroRNA29a regulates IL-33-mediated tissue remodelling in tendon disease. Nat Commun. 2015;6:6774.

20. Zhang M, Gong W, Zuo B, Chu B, Tang Z, Zhang Y, et al. The microRNA miR-33a suppresses IL-6-induced tumor progression by binding Twist in gallbladder cancer. Oncotarget. 2016;7(48):78640-78652.

21. Maurer B, Stanczyk J, Jungel A, Akhmetshina A, Trenkmann $M$, Brock M, et al. MicroRNA-29, a key regulator of collagen expression in systemic sclerosis. Arthritis Rheum. 2010;62(6): 1733-1743.

22. Padulo J, Oliva F, Frizziero A, Maffulli N. Muscles, Ligaments and Tendons Journal - Basic principles and recommendations in clinical and field science research: 2016 update. MLTJ. 2016;6(1):1-5.

23. Cook JL, Feller JA, Bonar SF, Khan KM. Abnormal tenocyte morphology is more prevalent than collagen disruption in asymptomatic athletes' patellar tendons. J Orthop Res. 2004;22(2):334-338.

24. Beederman M, Lamplot JD, Nan G, Wang J, Liu X, Yin L, et al. BMP signaling in mesenchymal stem cell differentiation and bone formation. J Biomed Sci Eng. 2013;6(8A):32-52. 
25. Legerlotz K, Jones ER, Screen HR, Riley GP. Increased expression of IL-6 family members in tendon pathology. Rheumatology (Oxford). 2012;51(7):1161-1165.

26. Lui PP, Chan LS, Cheuk YC, Lee YW, Chan KM. Expression of bone morphogenetic protein-2 in the chondrogenic and ossifying sites of calcific tendinopathy and traumatic tendon injury rat models. J Orthop Surg Res. 2009;4:27.

27. Xia J, Benner MJ, Hancock RE. NetworkAnalyst-integrative approaches for protein-protein interaction network analysis and visual exploration. Nucleic Acids Res. 2014;42(Web Server issue):W167-174.

28. Xia J, Gill EE, Hancock RE. NetworkAnalyst for statistical, visual and network-based meta-analysis of gene expression data. Nat Protoc. 2015;10(6):823-844.

29. Bartel DP. MicroRNAs: target recognition and regulatory functions. Cell. 2009;136(2):215-233.

30. Tanaka T, Narazaki M, Masuda K, Kishimoto T. Regulation of IL-6 in Immunity and Diseases. Adv Exp Med Biol. 2016;941: 79-88.

31. Castro-Villegas C, Perez-Sanchez C, Escudero A, Filipescu I, Verdu M, Ruiz-Limon P, et al. Circulating miRNAs as potential biomarkers of therapy effectiveness in rheumatoid arthritis patients treated with anti-TNFalpha. Arthritis Res Ther. 2015; 17:49.

32. Ruan W, Xu JM, Li SB, Yuan LQ, Dai RP. Effects of down-regulation of microRNA-23a on TNF-alpha-induced endothelial cell apoptosis through caspase-dependent pathways. Cardiovasc Res. 2012;93(4):623-632.

33. Zhang Y, Zhang B, Zhang A, Li X, Liu J, Zhao J, et al. IL-6 upregulation contributes to the reduction of miR-26a expression in hepatocellular carcinoma cells. Braz J Med Biol Res. 2013;46(1):32-38.

34. Luna C, Li G, Qiu J, Epstein DL, Gonzalez P. Role of miR-29b on the regulation of the extracellular matrix in human trabecular meshwork cells under chronic oxidative stress. Mol Vis. 2009;15:2488-2497.

35. Yu J, Luo H, Li N, Duan X. Suppression of Type I Collagen Ex- pression by miR-29b Via PI3K, Akt, and Sp1 Pathway, Part II: An In Vivo Investigation. Invest Ophthalmol Vis Sci. 2015;56 (10):6019-6028.

36. Abonnenc M, Nabeebaccus AA, Mayr U, Barallobre-Barreiro J, Dong $X$, Cuello $F$, et al. Extracellular matrix secretion by cardiac fibroblasts: role of microRNA-29b and microRNA-30c. Circ Res. 2013;113(10):1138-1147.

37. Ogawa T, lizuka M, Sekiya Y, Yoshizato K, Ikeda K, Kawada $\mathrm{N}$. Suppression of type I collagen production by microRNA$29 \mathrm{~b}$ in cultured human stellate cells. Biochem Biophys Res Commun. 2010;391(1):316-321.

38. Zhang K, Asai S, Hast MW, Liu M, Usami Y, Iwamoto M, et al Tendon mineralization is progressive and associated with deterioration of tendon biomechanical properties, and requires BMP-Smad signaling in the mouse Achilles tendon injury model. Matrix Biol. 2016;52-54:315-324.

39. Long J, Badal SS, Wang Y, Chang BH, Rodriguez A, Danesh FR. MicroRNA-22 is a master regulator of bone morphogenetic protein-7/6 homeostasis in the kidney. J Biol Chem. 2013;288(51):36202-36214.

40. Houbaviy HB, Murray MF, Sharp PA. Embryonic stem cellspecific MicroRNAs. Dev Cell. 2003;5(2):351-358.

41. Suh MR, Lee Y, Kim JY, Kim SK, Moon SH, Lee JY, et al. Human embryonic stem cells express a unique set of microRNAs. Dev Biol. 2004;270(2):488-498.

42. Smirnova L, Grafe A, Seiler A, Schumacher S, Nitsch R, Wulczyn FG. Regulation of miRNA expression during neural cel specification. Eur J Neurosci. 2005;21(6):1469-1477.

43. Thankam FG, Boosani CS, Dilisio MF, Agrawal DK. MicroRNAs associated with inflammation in shoulder tendinopathy and glenohumeral arthritis. Mol Cell Biochem. 2017.

44. Thankam FG, Boosani CS, Dilisio MF, Dietz NE, Agrawal DK MicroRNAs Associated with Shoulder Tendon Matrisome Disorganization in Glenohumeral Arthritis. PLoS One. 2016;11 (12): $\mathrm{e} 0168077$

45. Watts AE, Millar NL, Platt J, Kitson SM, Akbar M, Rech R, et al. MicroRNA29a Treatment Improves Early Tendon Injury. Mol Ther. 2017;25(10):2415-2426. 